\title{
MHD Unsteady Memory Convective Flow through Porous Medium with Variable Suction
}

\author{
S. A. Hussaini ${ }^{1 \dagger}$, M.V. Ramana Murthy ${ }^{2}$, A.Waheedullah $^{3}$ and Rafiuddin ${ }^{4}$ \\ ${ }^{I}$ Muffakham Jah college of Engineering, Hyderabad, Andhra Pradesh, 500034, India. \\ ${ }^{2}$ Osmania University, Hyderabad, Andhra Pradesh, 500007, India. \\ ${ }^{3}$ Maulana Azad National Urdu University, Hyderabad, Andhra Pradesh,500032, India \\ ${ }^{4}$ CVR college of Engineering, R.R Dist. Andhra Pradesh, 501510, India \\ $†$ Corresponding Author Email: amanullahsd@gmail.com
}

(Received April 5, 2011; accepted October 19, 2011)

\begin{abstract}
A free convective unsteady visco-elastic flow through porous medium of variable permeability bounded by an infinite vertical porous plate with variable suction, constant heat flux under the influence of transverse uniform magnetic field has been investigated in the present study. The permeability of porous medium fluctuates with time about the constant mean. Approximate solutions for mean velocity, transient velocity, mean temperature and transient temperature of non-Newtonian flow and skin friction are obtained. The effects of various parameters such as $\mathrm{P}_{\mathrm{r}}$ (Prandtl number), $\mathrm{G}_{\mathrm{r}}$ (Grashof number), $\mathrm{M}$ (Hartmann number), $\omega$ (frequency parameter) and $\mathrm{k}_{0}$ (mean permeability parameter) on the above are depicted, skin friction, amplitude and phase are shown graphically and discussed. Expressions for fluctuating parts of velocity ' $\mathrm{M}_{\mathrm{r}}$ ' and ' $\mathrm{M}_{\mathrm{i}}$ ' are found and plotted graphically, effects of different parameters on them are discussed.
\end{abstract}

Keywords: Free convection, Walter's liquid 'B', Variable permeability, Porous medium and Variable suction.

\section{INTRODUCTION}

In recent years, the problem of free convection flow through a porous medium of variable permeability has attracted the attention of a number of scholars because of its possible application in design of steam displacement process in an oil recovery and various geothermal systems. In fact a porous material containing the fluid is non- homogenous and there can be numerous in homogeneities present in the porous medium. Therefore, the permeability of porous medium may not necessarily be constant. This is studied extensively by Chanderasekhara et al. (1984), Vedha Nayagam et al. (1987) Singh et al. (1989). Sreekanth et al. (1996) studied the hydro magnetic free convective flow through a porous medium with variable permeability. Singh and Kumar (2002) have discussed three dimensional free convective flow and heat transfer through porous medium with periodic permeability. Later, Vershney and Singh (2005) extended the above problem for mass transfer. Sharma et al. (2006) studied MHD fluctuating free convective flow with radiation embedded in porous medium having variable permeability and heat source / sink. Mahanti and Gaur (2009) have studied the effects of varying viscosity and thermal conductivity on steady free convective flow and heat transfer along an isothermal vertical plate in the presence of heat sink. Anjali and Ganga (2010) have studied the dissipation effects on MHD non linear flow and heat transfer past a porous surface with prescribed heat flux. Humera et al. (2010) have studied the hydromagnetic free convective Revlin-Ericksen flow through a porous medium with variable permeability. Suneetha et al. (2011) studied the radiation and mass transfer effects on MHD free convective dissipative fluid in the presence of heat source / sink. Later Vasu et al. (2011) studied the radiation and mass transfer effects on transient free convection flow of a dissipative fluid past semi-infinite vertical plate with uniform heat and mass flux. Prasad et al. (2011) have studied the finite difference analysis of radioactive free convection flow past an impulsively started vertical plate with variable heat and mass flux. Seth et al. (2011) studied the effects of rotation and magnetic field on unsteady coquette flow in a porous channel. Singh and Kumar (2011) have studied the fluctuating heat and mass transfer on unsteady MHD free convection flow of radiating and reacting fluid past a vertical porous plate in slip-flow regime. Das et al. (2011) have studied the mass transfer effects on unsteady hydro magnetic convective flow past a vertical porous plate in a porous medium with heat source. Reddy and Reddy (2011) studied the mass transfer and heat generation effects on MHD free convective flow past an inclined vertical surface in a porous medium.

The aim of the authors is to extend the problem of Maharshi and Tak (2000) to memory fluid i.e Walter's liquid model B' $(1960,1962)$ with variable suction. The mixture of polymethyl mehacrylate and pyridine at $25^{\circ}$ 
C containing $30.5 \mathrm{~g}$ of polymer per liter behaves very nearly as the above mentioned liquid.

\section{Formulation Of The Problem}

We consider the flow of convective memory fluid through a porous medium bounded by an infinite vertical porous plate with constant heat flux under the influence of uniform transverse magnetic field. The $\mathrm{x}$ axis is taken along the plate in the upward direction and y - axis normal to it. All the fluid properties are assumed to be constant, except that influence of the density variations with temperature is considered only in the body force term. The magnetic field of small intensity $\mathrm{HO}$ is induced in the $\mathrm{y}$ - direction. Since the fluid is slightly conducting, the magnetic Reynolds number is far lesser than unity hence the induced magnetic field is neglected in comparison with the applied magnetic field following Sparrow and Cess (1961). The viscous dissipation and Darcy's dissipation terms are neglected for small velocities following Rudraiah et al. (1979). The flow in the medium is entirely due to buoyancy force. So under these conditions the flow with variable suction is governed by the following equations:

$\mathrm{v}=-\mathrm{v}_{0}\left(1+\varepsilon \mathrm{e}^{\mathrm{i} \omega \mathrm{t}}\right)$

$\frac{\partial u}{\partial t}+v \frac{\partial u}{\partial y}=g \beta_{1}\left(T-T_{\infty}\right)+v \frac{\partial^{2} u}{\partial y^{2}}-\frac{v u}{k(t)}-$

$\beta\left(\frac{\partial^{3} u}{\partial t \partial y^{2}}+v \frac{\partial^{3} u}{\partial y^{3}}\right)-\left(\frac{\sigma \mu e^{2} H_{0}^{2}}{\rho}\right) u$

$\frac{\partial T}{\partial t}+v \frac{\partial T}{\partial y}=\frac{\kappa}{\rho C_{p}} \frac{\partial^{2} T}{\partial y^{2}}$

The boundary conditions are:

$$
\begin{aligned}
& y=0: u=0, \frac{\partial T}{\partial y}=\frac{-q}{\kappa} \\
& y \rightarrow \infty: u=0, T=T_{\infty}
\end{aligned}
$$

where $\mathrm{u}$ and $\mathrm{v}$ are velocity components along $\mathrm{x}$ and $\mathrm{y}$ directions, $\omega$ is the frequency parameter $\beta_{1}$ is the coefficient of volume expansion, $\beta$ is kinematic viscoelasticity, $\sigma$ is electrical conductivity, $\mu_{e}$ is magnetic permeability, $\mathrm{H}_{0}$ is magnetic intensity, $k$ is thermal conductivity, $\mathrm{C}_{\mathrm{p}}$ specific heat at constant pressure, $\mathrm{q}$ is heat flux and the permeability of porous medium which is assumed to be of the form following Singh et al. (1989).

$$
k(t)=k_{0}\left(1+\varepsilon e^{i \omega t}\right)
$$

where $k_{0}$ is the mean permeability of the medium, $\omega$ is the frequency of fluctuation, $t$ the time and $(\varepsilon<<1)$ is a constant quantity, other symbols have usual meanings.

\section{Method Of SOlution}

Introducing the following non-dimensional quantities:

$$
\begin{aligned}
& y^{\prime}=\frac{y v_{0}}{\vartheta}, t^{\prime}=\frac{t v_{0}^{2}}{4 \vartheta}, \omega^{\prime}=\frac{4 \vartheta \omega}{v_{0}^{2}}, u^{\prime}=\frac{u}{v_{0}} \\
& G_{r}=\frac{g \beta_{1} q \vartheta^{2}}{k v_{0}^{4}}, P_{r}=\frac{\mu C_{p}}{\kappa}, R_{m}=\frac{\beta v_{0}^{2}}{\vartheta^{2}}, \\
& k_{0}^{\prime}=\frac{k_{0} v_{0}^{2}}{\vartheta^{2}}, M=\frac{\sigma \mu e^{2} H_{0}^{2} \vartheta}{v_{0}^{2} \rho}, \theta=\frac{\left(T-T_{\infty}\right) \kappa v_{0}}{q \vartheta}
\end{aligned}
$$

the Eq. (2) and Eq. (3) in view of Eq.(5) and Eq. (6) become after suppressing primes,

$$
\begin{aligned}
& \left(\frac{1}{4}\right) \frac{\partial u}{\partial t}-\left(1+\varepsilon e^{i \omega t}\right) \frac{\partial u}{\partial y} \\
& =G_{r} \theta+\frac{\partial^{2} u}{\partial y^{2}} \\
& -R_{m}\left[\left(\frac{1}{4}\right) \frac{\partial^{3} u}{\partial t \partial y^{2}}-\left(1+\varepsilon e^{i \omega t}\right) \frac{\partial^{3} u}{\partial y^{3}}\right] \\
& -\frac{u}{k_{0}}\left(1+\varepsilon e^{i \omega t}\right) \\
& -M u
\end{aligned}
$$$$
\left(\frac{1}{4}\right) \frac{\partial \theta}{\partial t}-\left(1+\varepsilon e^{i \omega t}\right) \frac{\partial \theta}{\partial y}=\left(\frac{1}{P_{r}}\right) \frac{\partial^{2} \theta}{\partial y^{2}}
$$

The corresponding boundary conditions are:

$$
\begin{aligned}
& y=0: u=0, \frac{\partial \theta}{\partial y}=-1 \\
& y \rightarrow \infty: u=0, \theta=0
\end{aligned}
$$

The partial differential equations, Eq. (7) and Eq. (8) are reduced to ordinary differential equations by assuming the following series expressions for velocity and temperature fields.

$$
\begin{aligned}
& u(y, t)=u_{0}(y)+\varepsilon e^{i \omega t} u_{1}(y)+\ldots \\
& \theta(y, t)=\theta_{0}(y)+\varepsilon e^{i \omega t} \theta_{1}(y)+\ldots
\end{aligned}
$$

substituting Eq. (10) and Eq. (11) in Eq. (7) and Eq. (8) and equating the coefficients of like powers of $\varepsilon$ to zero and neglecting $O\left(\varepsilon^{2}\right)$, the following set of ordinary differential equations are obtained :

$$
R_{m} u_{0}^{\prime \prime \prime}+u_{0}^{\prime \prime}+u_{0}^{\prime}-\left(\frac{1}{k_{0}}+M\right) u_{0}=-G_{r} \theta_{0}
$$

$$
R_{m} u_{1}^{\prime \prime \prime}+
$$$$
\left[\left(1-\frac{i R_{m} \omega}{4}\right) u_{1}^{\prime \prime}+u_{1}^{\prime}-\left(\frac{i \omega}{4}+\frac{1}{k_{0}}+M\right)\right] u_{1}^{\prime}
$$$$
=-G_{r} \theta_{1}-\frac{u_{0}}{k_{0}}-u_{0}^{\prime}
$$

$$
-R_{m} u_{0}^{\prime \prime \prime}
$$




$$
\begin{aligned}
& \frac{\theta_{0}^{\prime \prime}}{P_{r}}+\theta_{0}^{\prime}=0 \\
& \frac{\theta_{1}^{\prime \prime}}{P_{r}}+\theta_{1}^{\prime}=\frac{i \omega \theta_{1}}{4}=-\theta_{0}^{\prime}
\end{aligned}
$$

The corresponding boundary conditions are:

$$
\begin{aligned}
& y=0: u_{0}=0, u_{1}=0, \theta_{0}^{\prime}=-1, \theta_{1}^{\prime}=0 \\
& y \rightarrow \infty: u_{0}=0, u_{1}=0, \theta_{0}=0, \theta_{1}=0
\end{aligned}
$$

Equation (12) and Eq. (13) are third order differential equations when $R_{m} \neq 0$ and we have two boundary conditions, so we follow Beard and Walter's (1964),

$$
\begin{aligned}
& u_{0}=u_{01}+R_{m} u_{02}+O R_{m}^{2} \\
& u_{1}=u_{11}+R_{m} u_{12}+O R_{m}^{2}
\end{aligned}
$$

substituting these Eq. (17) and Eq. (18) into Eq. (12), equating different powers of $R_{m}$ and neglecting $\mathrm{O}\left(\mathrm{R}_{\mathrm{m}}{ }^{2}\right)$, we get

$$
\begin{aligned}
& u_{01}^{\prime \prime}+u_{01}^{\prime}-\left(\frac{1}{k_{0}}+M\right) u_{01}=-G_{r} \theta_{0} \\
& u_{11}^{\prime \prime}+u_{11}^{\prime}-\left(\frac{i \omega}{4}+\frac{1}{k_{0}}+M\right) u_{11} \\
& =\frac{-u_{01}}{k_{0}}-u_{01}^{\prime}-G_{r} \theta_{1} \\
& u_{01}^{\prime \prime \prime}+u_{02}^{\prime \prime}+u_{02}-\left(\frac{1}{k_{0}}+M\right) u_{02}=0 \\
& u_{11}^{\prime \prime \prime}-\frac{i \omega}{4} u_{11}^{\prime \prime}+u_{12}^{\prime \prime}+u_{12}^{\prime}-\left(\frac{i \omega}{4}+\frac{1}{k_{0}}+M\right) u_{12} \\
& =\frac{-u_{02}}{k_{0}}-u_{02}^{\prime}-u_{01}^{\prime \prime \prime}
\end{aligned}
$$

The boundary conditions are:

$$
\begin{aligned}
& y=0: u_{01}=u_{02}=u_{11}=u_{12}=0 \\
& y \rightarrow \infty: u_{01}=u_{02}=u_{11}=u_{12}=0
\end{aligned}
$$

The velocity and temperature fields are given by

$$
\begin{aligned}
& u=u_{0}+\varepsilon u_{1}=u_{01}+R_{m} u_{02} \\
& +\varepsilon\left(u_{11}+R_{m} u_{12}\right) \\
& \theta=\theta_{0}+\varepsilon \theta_{1}
\end{aligned}
$$

Taking real part of solution for the velocity field and temperature field they can be expressed in terms of fluctuating part as

$$
\begin{aligned}
& u(y, t)=u_{0}(y)+ \\
& \varepsilon\left(M_{r} \cos \omega t-M_{i} \sin \omega t\right) \\
& \theta=\theta_{0}(y)+\varepsilon\left(N_{r} \cos \omega t-N_{i} \sin \omega t\right)
\end{aligned}
$$

Where

$$
M_{r}+i M_{i}=u_{1}(y)
$$

$N_{r}+i N_{i}=\theta_{1}(y)$

The expressions of transient velocity and transient temperature for $\omega t=\pi / 2$ are given by

$u\left(y, \frac{\pi}{2 \omega}\right)=u_{0}(y)-\varepsilon M_{i}$

$\theta\left(y, \frac{\pi}{2 \omega}\right)=\theta_{0}(y)-\varepsilon N_{i}$

The skin friction at the plate in terms of amplitude and phase is:

$C_{f}=\frac{T_{\omega}}{\rho v_{0}^{2}}=\left(\frac{\partial u}{\partial y}\right)_{y=o}$

$=\frac{G_{r}}{P_{1}}\left(P_{r}-r_{1}\right)\left(1+R_{m} P_{3}\right)$

$+R_{m} r_{3}$

$+\varepsilon|\lambda| \cos (\omega t+\alpha)$

Where

$$
\begin{aligned}
& C_{f}=\frac{T_{\omega}}{\rho v_{0}^{2}}=\left(\frac{\partial u}{\partial y}\right)_{y=o} \\
& =\frac{G_{r}}{P_{1}}\left(P_{r}-r_{1}\right)\left(1+R_{m} P_{3}\right) \\
& +R_{m} r_{3} \\
& +\varepsilon|\lambda| \cos (\omega t+\alpha)
\end{aligned}
$$

Where

$$
\lambda_{r}+i \lambda_{i}=\lambda \text { and } \tan \alpha=\frac{\lambda_{r}}{\lambda_{i}}
$$

\section{DiscuSsion AND CONCLUSIONS}

The (thermal Grashoff number) $G_{r}$ signifies the relative effect of the thermal buoyancy to the viscous hydrodynamic force, so the rise in mean velocity is due to enhancement of thermal buoyancy force. In the neighborhood of plate steep rise is noted then gradually diminishing. From Eq. (6), we see that mean permeability parameter is in direct proportion to mean permeability enhancement of mean velocity is attributed to higher values of mean permeability parameter. (Prandtle number) $\mathrm{P}_{\mathrm{r}}$ defined by the relative ratio of kinematics viscosity to thermal diffusivity viscous forces tend to slow down the velocity so reduction of mean velocity is due to increase in Prandtle number value. Application of transverse magnetic field gives rise to Lorentz force, which is resistive typed force analogous to drag force, higher values of magnetic field suppresses mean velocity. Transient velocity profiles are influenced by mean velocity and fluctuating part $\mathrm{M}_{\mathrm{i}}$ as it contains both in its expressions. Transitive velocities are negative buoyancy force, Lorentz force and viscous force are likely to suppress the transient velocity parameter profiles whereas higher 
values of mean permeability parameter $\mathrm{k}_{0}$ and frequency parameter $\omega$ are influencing the same refer Fig. 1. and Fig. 2.

The dominant viscous forces bring down degree of hotness so the effect of Prandtle number is to subside the temperature distribution in thermal boundary layer refer Fig. 3 and Fig. 4.

Fluctuating parts $\mathrm{M}_{\mathrm{r}}$ and $\mathrm{M}_{\mathrm{i}}$ are negative buoyancy force and viscous forces tends to reduce the fluctuating parts $\mathrm{M}_{\mathrm{r}}$ and $\mathrm{M}_{\mathrm{i}}$ considerably whereas mean

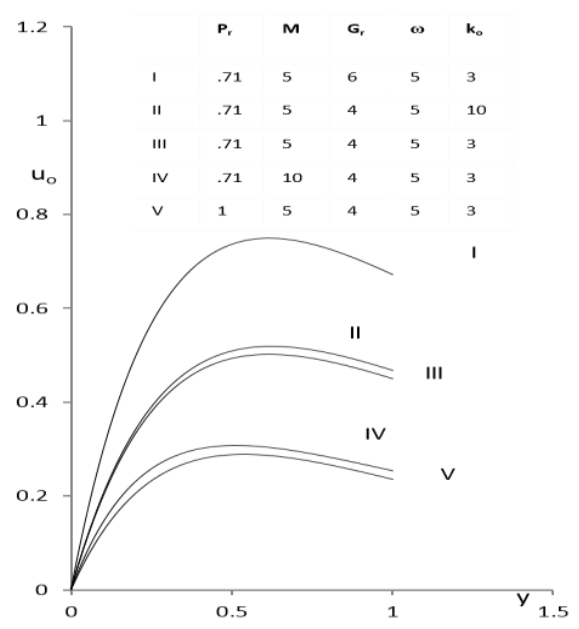

Fig. 1. Effect of $k_{0}, M, G_{r}, \omega, P_{r}$ on mean velocity $u_{0}$ versus y for $\mathrm{R}=0.05$

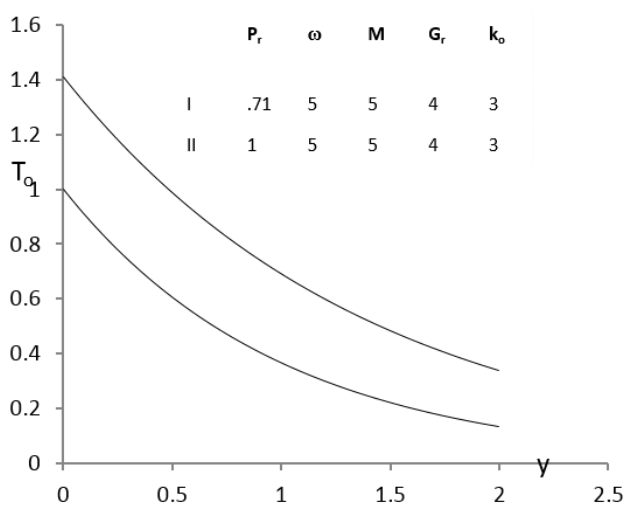

Fig. 3. Effect of $k_{0}, M, G_{r}, \omega, P_{r}$ on mean temperature $T_{0}$ versus $y$ for $\mathrm{R}=0.05$ and $\omega \mathrm{t}=\pi / 2$ permeability parameter and frequency parameter have same effect on $M_{r}$ and $M_{i}$ refer Fig. 5. and Fig. 6.

As expected amplitude is positive buoyancy force. Lorentz force, kinematic viscous force and mean permeability enhancing amplitude the less the frequency the more is amplitude is refer Fig.7. The phase is positive and the effects of $P_{r}, G_{r}$ and $M$ are to influence the phase that means there is a phase lead refer Fig. 8.

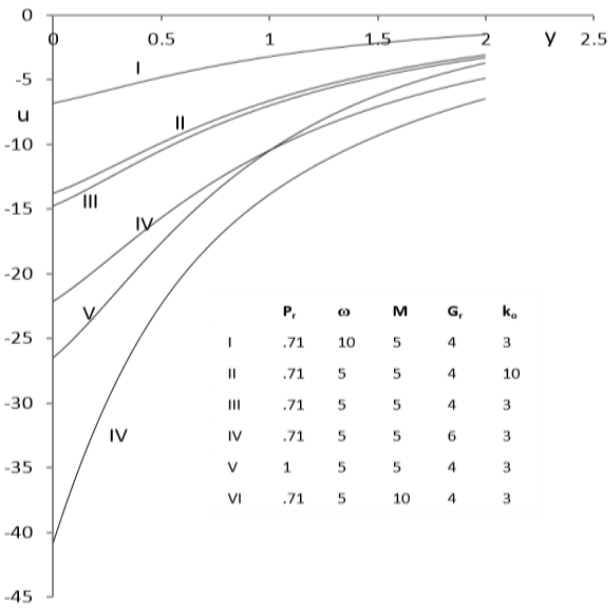

Fig. 2. Effect of $k_{0}, M, G_{r}, \omega, P_{r}$ on transient velocity $u$ versus $\mathrm{v}$ for $\mathrm{R}=0.05$ and $\omega \mathrm{t}=\pi / 2$

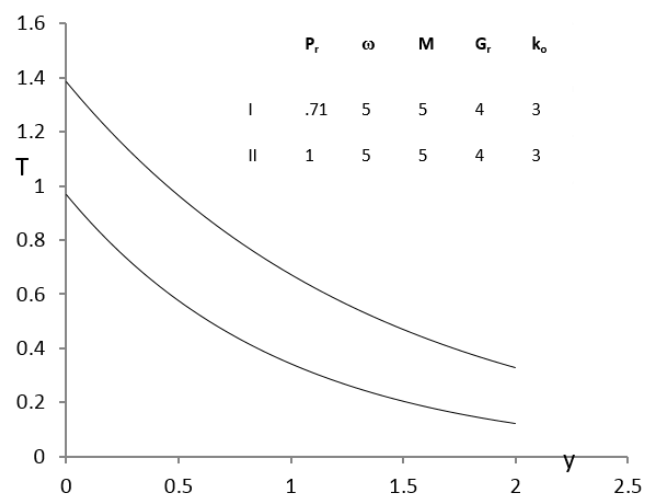

Fig. 4. Effect of $\mathrm{k}_{0}, \mathrm{M}, \mathrm{G}_{\mathrm{r}}, \omega, \mathrm{P}_{\mathrm{r}}$ on mean temperature $\mathrm{T}$ versus $\mathrm{y}$ for $\mathrm{R}=0.05$ 


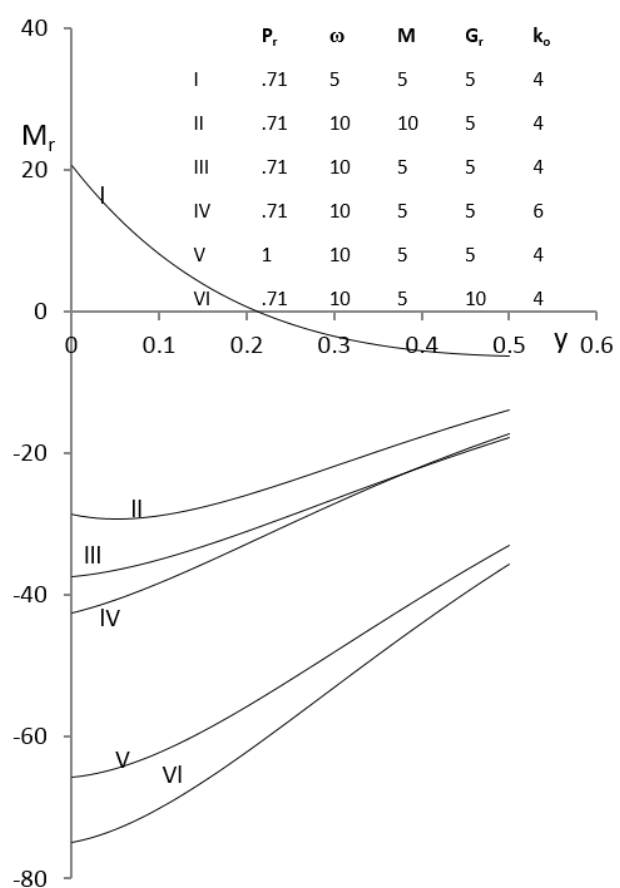

Fig. 5. Fluctuating parts of velocity profile $M_{r}$

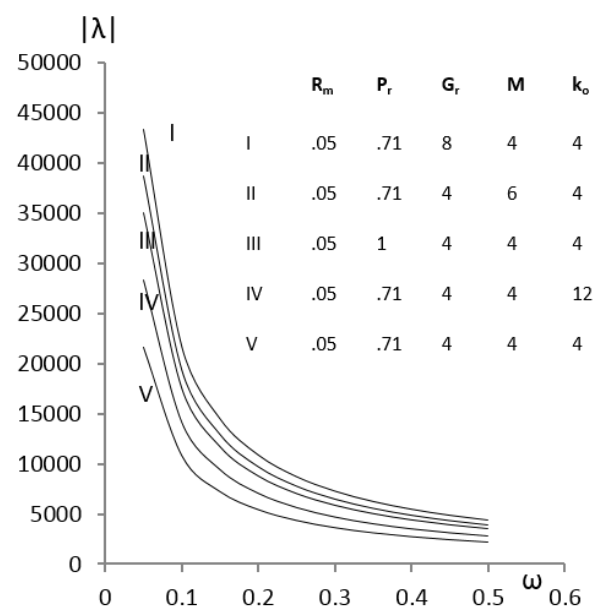

Fig. 7. Amplitude versus Frequency parameter $\omega$

\section{REFERENCES}

Anjali, S. P. and B. Ganga (2010). Dissipation effects on MHD non linear flow and heat transfer past a porous surface with prescribed heat flux. Journal of Applied Fluid Mechanics, 3(1), 1-6.

Beard, D. W. and K. Walters (1964). Elastico- viscous boundary-layer flows. I.Two-dimensional flow near a stagnation point Proc. Camb. Phil. Soc., 60(3), 667-674.

Chandrasekhara, B. C. and P. M. S. Namboodiri (1985). Influence of variable permeability on combined vertical surfaces in porous medium International Journal of Heat Mass Transfer, 28, 199-206.

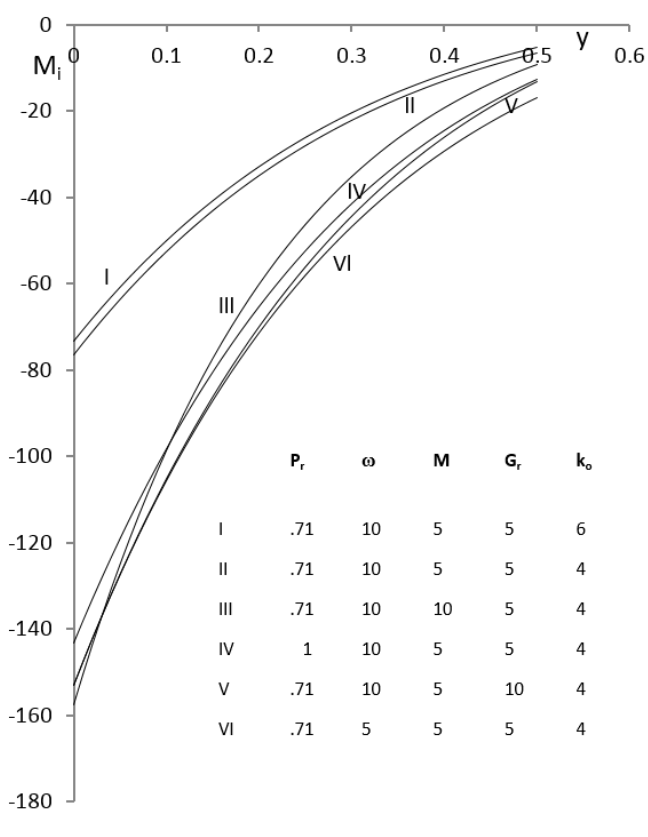

Fig. 6. Fluctuating parts of velocity profile $M_{i}$

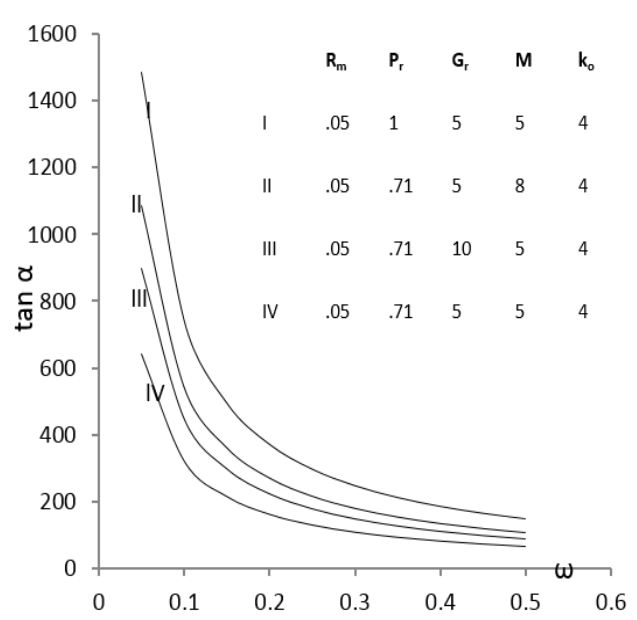

Fig. 8. Phase versus Frequency parameter $\omega$

Chandrashekar, B. C., P.M.S. Namboodri and A. R. Hanumanthappa (1984). Warmeuna Stoffubertragung 18, 17

Das, S. S., S. R. Biswal, U. K. Tripathy, and P. Das (2011). Mass transfer effects on unsteady hydro magnetic convective flow past a vertical porous plate in a porous medium with heat source. Journal of Applied Fluid Mechanics, 4(4), 91-100.

Humera, G. N., M. V. Ramana Murthy,M. C. K. Reddy, Rafiuddin, A. Ramu, and S. Rajender (2010). Hydromagnetic free convective Revlin-Ericksen flow through a porous medium with variable permeability International Journal of 
computational and Applied Mathematics, 5(3), 267-275.

Mahanti, N. C. and P. Gaur (2009). Effects of varying viscosity and thermal conductivity on steady free convective flow and heat transfer along an isothermal vertical plate in the presence of heat sink. Journal of Applied Fluid Mechanics, 2(1), 23-28.

Maharshi, A. and S. S. Tak (2000). Fluctuating free convection through porous medium due to infinite vertical plate with constant heat flux. J. Indian Acad Math ,.22, 293.

Nayagam, M. V., P. Jain and G. Fairweather (1987). The effect of surface mass transfer on buoyancyinduced flow in a variable-porosity medium adjacent to a horizontal heated plate Int Comm Heat Mass Transfer 14, 495.

Prasad. V.R., N. B. Reddy, R. B. Vasu (2011). Finite difference analysis of radioactive free convection flow past an impulsively started vertical plate with variable heat and mass flux. Journal of Applied Fluid Mechanics, 4(1), 59-68.

Reddy, M. G. and N. B. Reddy (2011). Mass transfer and heat generation effects on MHD free convective flow past an inclined vertical surface in a porous medium. Journal of Applied Fluid Mechanics, 4(2), 7-11.

Rudraiah, N., B. C. Chandrasekhar, R. Veerabhadraiah, and S. T. Nagaraj (1979). Some flow problems in porous media PGSAM Ser., 2, 13-16.

Seth, G. S., M. S. Ansari and R. Nandkeolyar (2011). Effects of rotation and magnetic field on unsteady coquette flow in a porous channel. Journal of Applied Fluid Mechanics, 4(2), 95-103.

Sharma, B., M. Agarwal and R. C. Chaudhary (2006). MHD fluctuating free convective flow with radiation embedded in porous medium having variable permeability and heat source/sink J.Tech.Physics, 47(1).

Singh, K. D., and R. Kumar (2011). Fluctuating heat and mass transfer on unsteady MHD free convection flow of radiating and reacting fluid past a vertical porous plate in slip-flow regime. Journal of Applied Fluid Mechanics, 4(4), 101-106.
Singh, K. D. and R. Sharma (2002). Three- dimensional free convective flow and heat transfer through a porous medium with periodic permeability. Indian J. Pure appl. Math. 33 (6), 941-949.

Singh, P., J. K. Misra and K. A. Narayana (1989).Int. J. Numerical and Analytical Methods Geomechanics, 13,443 .

Sparrow, E. M. and R. D. Cess (1961, December). The effect of a magnetic field on free convection heat transfer International Journal of Heat and Mass Transfer, 3(4), 267-274.

Sreekanth, S., S. Venkataramana, and S. Ramakrishna (1996). Hydromagnetic free convective flow through a porous medium with variable permeability. Acta Ciencia Indica,.vol XXII (3), 267.

Suneetha. S., N. B. Reddy and V. R. Prasad (2011). Radiation and mass transfer effects on MHD free convective dissipative fluid in the presence of heat source / sink. Journal of Applied Fluid Mechanics, 4(1), 107-113.

Vasu. B, V. R. Prasad and N. B. Reddy (2011). Radiation and mass transfer effects on transient free convection flow of a dissipative fluid past semi-infinite vertical plate with uniform heat and mass flux. Journal of Applied Fluid Mechanics, $4(1), 15-26$.

Vershney, N. K. and D. Singh (Math Dep, S.V. Coll, Aligarh, U. P) (2005). Three dimensional free convective flow with heat and mass transfer through a porous medium with periodic permeability. Bull Calcutta math Soc, 97(2), 95106.

Walter, K. (1960). The Motion of Elastico -Viscous Liquids Contained Between Coaxial Cylinders Quart. Jour. Mech. Applied Math, 13, 444.

Walter, K. (1962). Non - Newtonian Effects in Some Elastico - viscous Liquids Whose Behavior At Some Rates of Shear is Characterized By General Equation of State Quat. Jour. Mech Applied Math., 15, 63-76. 